\title{
Critical Roles of the PI3K-Akt-mTOR Signaling Pathway in Apoptosis and Autophagy of Astrocytes Induced by Methamphetamine
}

https://doi.org/10.1515/chem-2019-0015

received June 18, 2018; accepted December 6, 2018.

Abstract: This study aimed to reveal potential roles of the phosphatidylinositol 3 kinase (PI3K)-protein kinase B (Akt)-mammalian target of rapamycin (mTOR) signaling pathway in apoptosis and autophagy of astrocytes induced by methamphetamine (METH). A Cell Counting Kit-8 (CCK-8) was used to determine the reduction in proliferation of U-118 MG cells induced by METH. Hoechst 33258 and flow cytometry were used to observe the astrocytes. Western blot analysis was performed to evaluate protein expression and phosphorylation levels. METH inhibited the proliferation of U-118 MG cells and induced apoptosis and autophagy. Western blot analysis showed that the ratio of LC3-II/I was increased, whereas the expression of $\mathrm{Bcl}-2$ was decreased. The phosphorylation cascade of kinases in the PI3K-Akt-mTOR signaling pathway was significantly inhibited by METH exposure, as were proteins downstream of mTORC1, such as p70s6k, rps6, 4EBP1 and eIF4E. METH inhibited proliferation of U-118 MG cells and induced apoptosis and autophagy. The PI3K-Akt-mTOR signaling pathway likely plays a critical role in these effects.

\footnotetext{
*Corresponding authors: Yu Shi, Guang-Hui Cui, Yun Chen, Shenzhen Key Laboratory for Drug Addiction and Medication Safety, Peking University Shenzhen Hospital, Shenzhen Peking UniversityThe Hong Kong University of Science and Technology Medical Center, Shenzhen City, Guangdong Province, China, 518036, E-mails:1714634835@qq.com; cuiguanghui175@aliyun.com; chenyun6308@139.com

Han-Qing Liu, A-Zhen Hu, Ming-Hua Li, Jue-Lian Wu, Li Liu: Shenzhen Key Laboratory for Drug Addiction and Medication Safety, Peking University Shenzhen Hospital, Shenzhen Peking UniversityThe Hong Kong University of Science and Technology Medical Center, Shenzhen City, Guangdong Province, China, 518036 Ya-Wen An: The State Key Laboratory of Chemical Oncogenomics, Key Laboratory of Chemical Genomics, School of Chemical Biology and Biotechnology, Peking University Shenzhen Graduate School, Shenzhen City, Guangdong Province, China, 518055
}

Keywords: methamphetamine; protein kinase B; astrocyte; apoptosis; autophagy.

\section{Introduction}

Drug abuse has increased significantly year by year, both in China and the rest of the world, and has now reached epidemic proportions. Synthetic drugs, including methamphetamine (METH), have become the most widely abused substances, with their use far exceeding that of opiates. METH abusers are increasingly recognized to suffer from sustained brain damage, leading to cognitive and motor deficits. Although the mechanisms contributing to this damage are not fully understood, neuronal cell death has been observed in many animal models following METH exposure. This cell death, which involves cells in reward circuits such as the hippocampus, nucleus accumbens and corpus striatum, may contribute to the pathogenesis of neurotoxicity [1-4].

Recently, METH-induced microglial activation and death have also been well documented [5-6], although the relative roles of signaling pathways in apoptosis and autophagy remain to be elucidated. The phosphatidylinositol 3 kinase (PI3K)-protein kinase B (Akt)-mammalian target of rapamycin (mTOR) signaling pathway regulates numerous biological processes, including cell survival, proliferation and growth, and glucose metabolism [7-9], and has been demonstrated to be involved in the neuronal adaptations that underlie drugrelated behaviors such as reward seeking and excessive drug intake [10]. It also has been reported that the PI3KAkt-mTOR signaling pathway is the critical factor for cueinduced reinstatement of drug seeking [11]. In a previous study, we found that METH could induce conditioned place preference (CPP) in rats, and that this effect was regulated by the PI3K-AKT-mTOR signaling pathway [13]. However, the roles of PI3K-Akt-mTOR signaling pathway in 
Table 1: Information of the reagents.

\begin{tabular}{|c|c|c|c|}
\hline Reagents & Abbreviation & Purity / cat.no & Source \\
\hline Cell Counting Kit-8 & CCK-8 & cat. no. CK04 & Dojindo, Tokyo, Japan \\
\hline IP Lysis Buffer & / & cat. no. 87788 & Thermo Fisher Scientific, Waltham, MA, USA \\
\hline Alexa Fluor ${ }^{\mathrm{TM}} 488$ Annexin V Kit & / & cat. no. V13241 & \\
\hline phenyl methane sulfonyl fluoride & PMSF & $\geq 98.5, \mathrm{HPLC}$ & \\
\hline Hoechst 33258 & / & $\geq 98, \mathrm{HPLC}$ & Beyotime, Shanghai, China \\
\hline $\mathrm{Na}_{3} \mathrm{VO}_{4}$ & l & 99.98, HPLC & Sigma-Aldrich, Burlington, MA, USA \\
\hline $\mathrm{NaF}$ & / & $\geq 99, \mathrm{HPLC}$ & \\
\hline phospho-phosphatidylinositol 3 kinase & $p$-PI3K & cat. no. PA5-17387 & Invitrogen, Carlsbad, CA, USA \\
\hline phospho-p70 ribosomal s6 kinase & $p$-p70s6k & cat. no. 701083 & \\
\hline phospho-ribosomal protein s6 & $p$-rps6 & cat. no. MA5-15140 & \\
\hline phospho-4E binding protein 1 & $p$-4EBP1 & cat. no. PA5-17728 & \\
\hline phospho-protein synthesis initiation factor $4 \mathrm{E}$ & $p$-elF4E & cat. no. PA5-17919 & \\
\hline BCL2-Associated X & Bax & cat. no. \#2772 & Cell Signaling Technology, Danvers, MA, USA \\
\hline B-cell lymphoma-2 & $\mathrm{Bcl}-2$ & cat. no. \#2782 & \\
\hline Light Chain 3 & LC 3 & cat. No. \#4108 & \\
\hline$\beta$-actin & l & cat. no. \#4970 & \\
\hline phospho-protein kinase B & $p$-Akt & cat. no. \#4060 & \\
\hline phospho-mammalian target of rapamycin & $p$-mTOR & cat. no. \#5536 & \\
\hline HRP-labeled Goat Anti-Rabbit IgG & / & cat. no. A0208, & Beyotime, Shanghai, China \\
\hline primary antibody dilution buffe & / & cat. no. T143195 & Biosharp, Hefei, China \\
\hline chemiluminescent HRP substrate & 1 & cat. no. WBKLS0100 & Millipore, MA, USA \\
\hline
\end{tabular}

regulating the survival and death of glial cells induced by METH were still unclear. Therefore, in the present study, we aimed to observe the inhibition of cell proliferation, apoptosis and autophagy of astrocytes induced by METH, and to reveal the role of the PI3K-Akt-mTOR signaling pathway. These studies might help us to understand the mechanisms of METH addiction and might provide some avenues for withdrawal treatment.

\section{Materials and methods}

\subsection{Cell culture and treatment}

METH (purity > 99\%) was provided by the compulsory isolation and detoxification program operated in Shenzhen, China. U-118 MG cells were provided by the Hong Kong University of Science and Technology and cultured in DMEM supplemented with 10\% FBS. All primary antibodies were diluted 1000 times with a primary antibody dilution buffer. Information regarding the reagents are summarized in Table. 1 . Cells were added to well-plates at a density of $1 \times 10^{6}$ cells $/ \mathrm{mL}$ and incubated at $37^{\circ} \mathrm{C}$ under an atmosphere containing $5 \% \quad \mathrm{CO}_{2}$. The concentrations of METH tested using the CCK-8 were 198, 296, 444, 667 and $1000 \mu \mathrm{g} / \mathrm{mL}$. Six replicate wells were set for each concentration, and identical cells without METH treatment were used as a control. A solution of METH in DMEM $(1000 \mu \mathrm{g} / \mathrm{mL})$ was progressively diluted after sterilization by filtration. Cells were exposed to METH for $24 \mathrm{~h}$ and CCK-8 reagent $(10 \mu \mathrm{L})$ was then added to the medium $(100 \mu \mathrm{L})$. The cells were cultured at $37^{\circ} \mathrm{C}$ for $1 \mathrm{~h}$ and the absorbance of the cells at $450 \mathrm{~nm}$, with a reference wavelength of $405 \mathrm{~nm}$, was then determined using a Spectra Max i3x Microplate Reader (Molecular Devices, Shanghai, China). The inhibition of cell proliferation at every concentration (IC) was calculated with the formula 
IC $(\%)=\left(A_{0}-A_{M}\right) / A_{0} \times 100$, wherein $A_{0}$ means the absorbance of the control group and $A_{M}$ represents the absorbance of groups treated with METH of different concentrations. A concentration-inhibition curve was fitted using the IC (\%) as the $\mathrm{Y}$-axis and concentrations of METH as the X-axis.

An Alexa Fluor ${ }^{\mathrm{TM}} 488$ Annexin V/Dead Cell Apoptosis Kit, together with flow cytometry (FCW), was used to detect the presence of apoptotic cells. The concentrations of METH were 198, 296, 444, 667 and $1000 \mu \mathrm{g} / \mathrm{mL}$, and identical cells without METH treatment were used as controls. After exposure to METH for $24 \mathrm{~h}$, the cells were collected and resuspended at a concentration of $1 \times 10^{6}$ cells/mL. Annexin V solution $(5 \mu \mathrm{L})$ and Alexa Fluor ${ }^{\mathrm{TM}}$ 488 solution $(5 \mu \mathrm{L})$ were added sequentially to the cell suspension $(1 \mathrm{~mL})$, and the mixture was then incubated in the dark at room temperature (RT) for $15 \mathrm{~min}$. An Accuri ${ }^{\circledR}$ C6 flow cytometer (BD Pharmingen) was then used to identify apoptotic cells. For negative control, only Annexin V solution ( $5 \mu \mathrm{L}$ ) or Alexa Fluor ${ }^{\mathrm{TM}} 488$ solution (5 $\mu \mathrm{L})$ was added.

Hoechst 33258 was dissolved in water $(1 \mathrm{mg} / \mathrm{mL})$ to provide a stock solution, which was diluted to $0.5 \mu \mathrm{g} / \mathrm{mL}$ for DNA staining. The cells were collected and fixed with ethyl acetate after exposure to METH for $24 \mathrm{~h}$. The cells were then counterstained with Hoechst 33258 for 15 min at RT and observed using an IX51 fluorescence microscope (Olympus, Tokyo, Japan). Hoechst 33258 is a specific fluorescent DNA probe and the nuclei showed bright blue fluorescence after staining.

\subsection{Western blot analysis}

Cell culture and treatment were performed as described above, using METH concentrations of 198, 296, 444, 667 and $1000 \mu \mathrm{g} / \mathrm{mL}$, and identical cells without METH treatment were used as control. After treatment with METH for $24 \mathrm{~h}$, the cells were collected, placed in 1.5-mL microtubes and treated with cell lysis buffer containing PMSF (1 mM), $\mathrm{Na}_{3} \mathrm{VO}_{4}(1 \mathrm{mM})$ and $\mathrm{NaF}(20 \mathrm{mM})$. After $30 \mathrm{~min}$ at $4^{\circ} \mathrm{C}$, the mixture of cells and lysis buffer was centrifuged at $12,000 \mathrm{~g}$ for $15 \mathrm{~min}$ at $4^{\circ} \mathrm{C}$ to separate the proteins. Loading buffer $(5 \times)$ was added to the protein solutions at a ratio of 1: 4 and the samples were digested for $10 \mathrm{~min}$ at $98^{\circ} \mathrm{C}$. Protein samples $(5 \mu \mathrm{L})$ were loaded into the wells of polyacrylamide gels and separated by electrophoresis at $80-120 \mathrm{~V}$. Proteins were transferred to polyvinylidene fluoride membranes with an electrical current of 0.33 A for $1.5 \mathrm{~h}$. The membranes were incubated overnight at $4^{\circ} \mathrm{C}$ with primary antibodies and then for $1 \mathrm{~h}$ at RT with a horseradish peroxidase (HRP)-labeled Goat Anti-Rabbit
IgG (1:2000). Protein expression and phosphorylation levels were determined using a $5220 \mathrm{~S}$ chemiluminescence detector (Tanon, Guangzhou, Guangdong, China) with immobilon $^{\mathrm{TM}}$ Western chemiluminescent HRP substrate.

\subsection{Statistical analysis}

Values for the target protein levels were normalized to $\beta$-actin and analyzed using Quantity One software (version 4.4.0; Bio-Rad, Hercules, CA, USA). Data were expressed as means \pm standard deviation (SD) and intergroup differences were compared by one-way ANOVA analysis, using SPSS 17.0 software (SPSS, Chicago, IL, USA). A probability $(p)$ value of $<0.05$ was considered to be statistically significant.

Ethical approval: The conducted research is not related to either human or animal use.

\section{Results}

\subsection{Inhibition of astrocyte proliferation and induction of apoptosis by METH}

The CCK- 8 test, Hoechst 33258 staining, and FCW were used to observe METH-induced inhibition of proliferation and induction of apoptosis in U-118 MG cells. METH inhibited cell proliferation in a dose-dependent manner, with a positive logarithmic correlation between the concentration and the degree of inhibition (Figure 1). Obvious apoptotic features, such as nuclear retraction, were observed after Hoechst 33258 fluorescence staining (Figure 2). The FCW test, using Alexa Fluor ${ }^{\mathrm{TM}} 488$ Annexin $\mathrm{V}$, indicated that METH dose-dependently induced cell apoptosis (Figure 3).

\subsection{Changes of protein expression and phos- phorylation levels after exposure to METH}

Bcl-2, an inner mitochondrial membrane protein, inhibits apoptotic neuronal cell death. METH inhibited the expression of Bcl-2; however, the expression of Bax showed no significant differences (Figure 4a). Autophagy, a type of programmed cell death that is independent of apoptosis, is negatively regulated by the mTORC1 signaling pathway. The LC3-II/I ratio is generally considered to be an indicator of the autophagosome, which is necessary for autophagy. Here, the LC3-II/I ratio was significantly increased after 


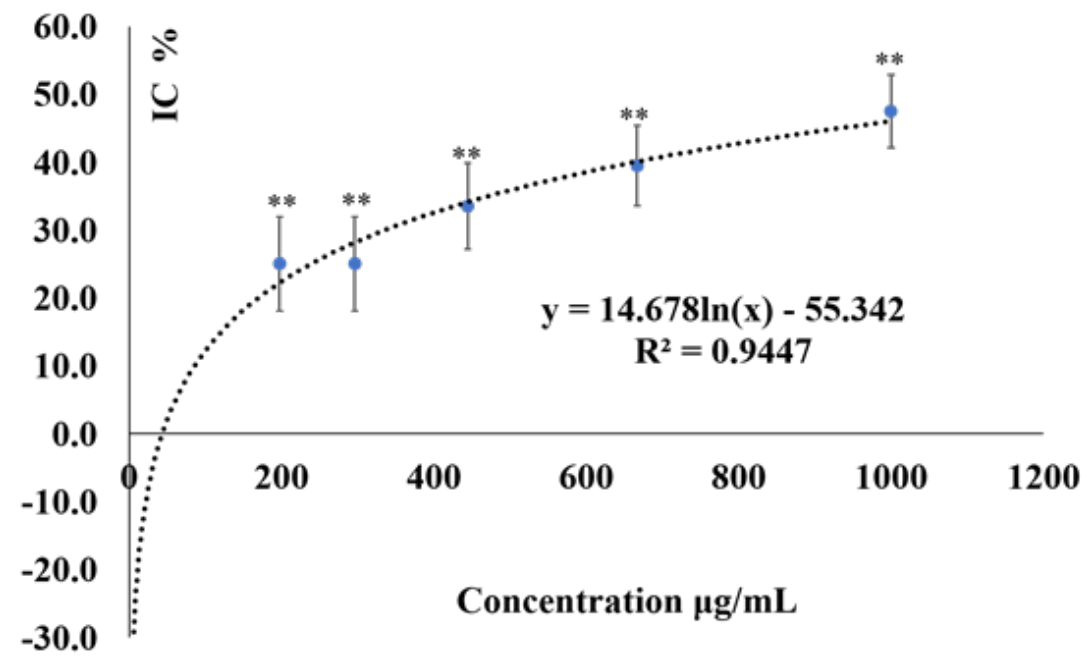

Figure 1: METH exerts dose-dependent inhibition of the proliferation of U-118 MG cells. The concentrations of METH were 198, 296, 444, 667 and $1000 \mu \mathrm{g} / \mathrm{mL}$, and a CCK-8 test was used to evaluate the cell proliferation. Data are presented as means \pm SD $(n=6)$, and the annotation ** indicates a $p$ value $<0.01$ versus control group.

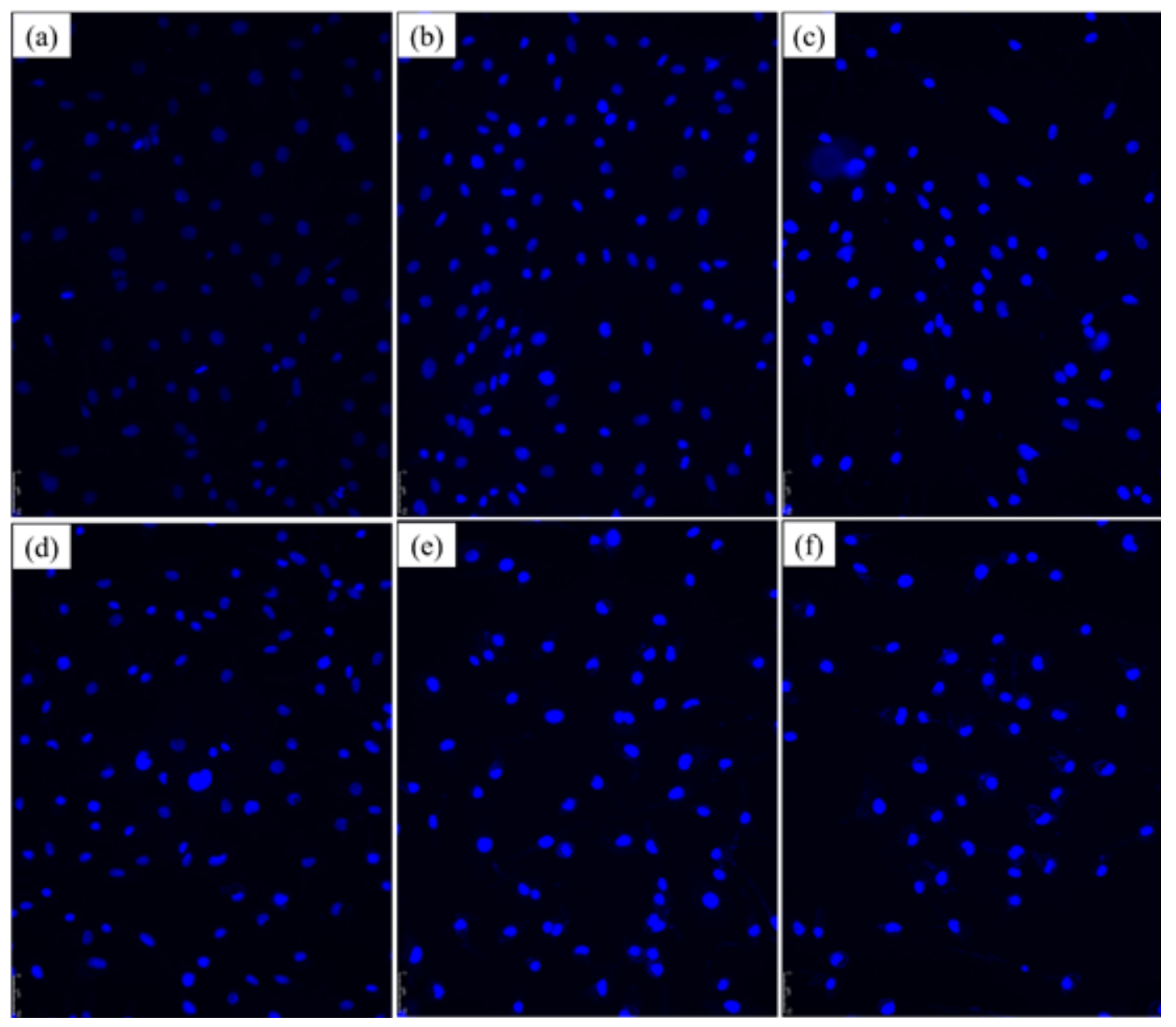

Figure 2: Immunocytochemical detection of METH induced apoptosis in U-118 MG cells. Hoechst 33258 staining indicated that METH induced U-118 MG cells apoptosis. Morphologic changes in nuclei were observed with Hoechst 33258 staining under fluorescence microscopy. (a) Control group. The cells were shaped normally and had round, intact nuclei; (b) METH $198 \mu \mathrm{g} / \mathrm{mL}$ group; (c) $\mathrm{METH} 296 \mu \mathrm{g} / \mathrm{mL}$ group; (d) METH $444 \mu \mathrm{g} / \mathrm{mL}$ group; (e) METH $667 \mu \mathrm{g} / \mathrm{mL}$ group group; (f) METH $1000 \mu \mathrm{g} / \mathrm{mL}$ group. Original magnification is $200 \times$. 


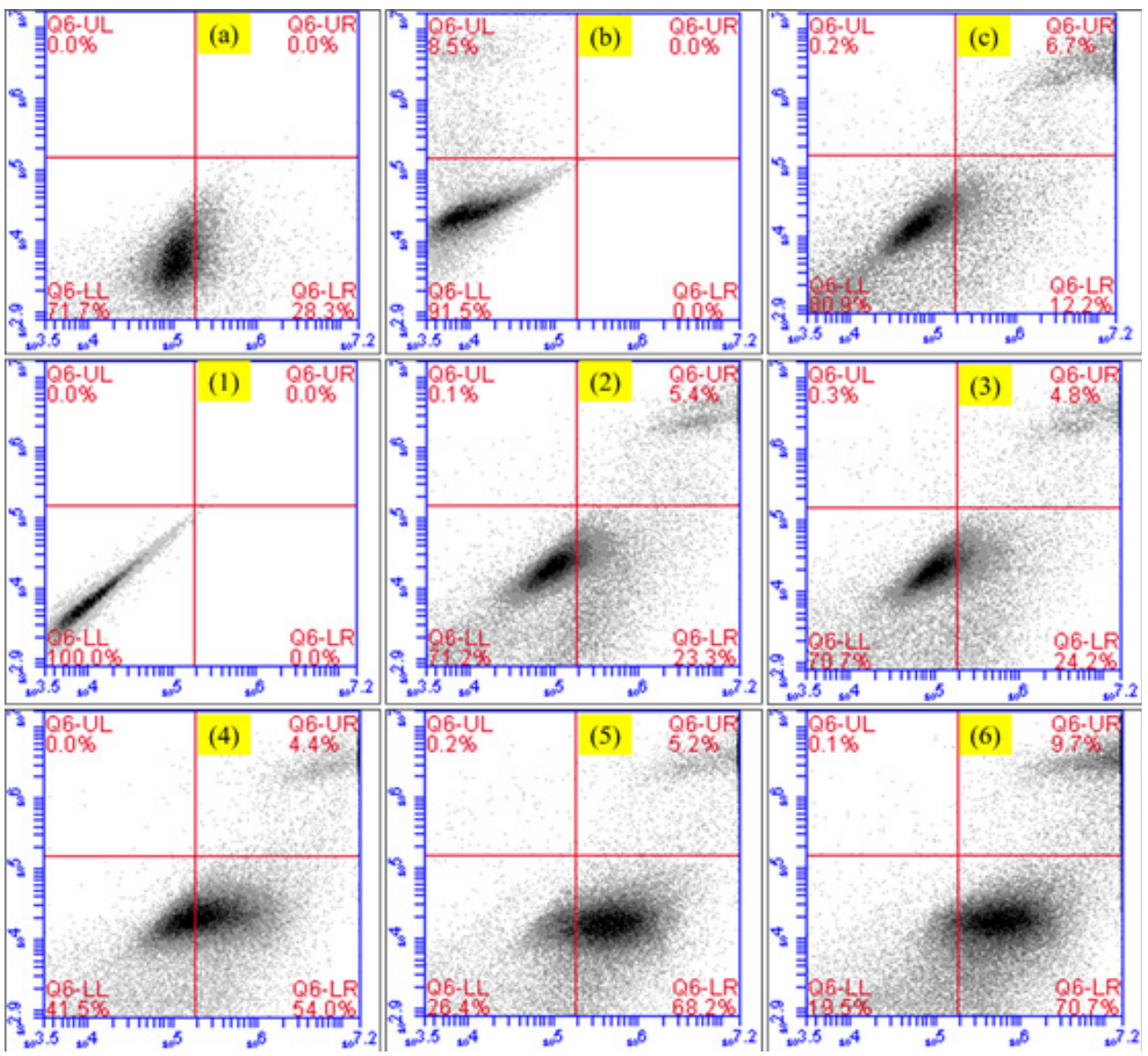

Figure 3: Cells were stained with Annexin V- Alexa Fluor ${ }^{\mathrm{TM}} 488$ for verifying the apoptotic or necrotic cell ratio. METH induced U-118 MG cells apoptosis. (a) Alexa Fluor ${ }^{\mathrm{TM}} 488$ positive control; (b) Annexin V positive control; (c) double staining positive control. (1) Natural group; (2) METH $198 \mu \mathrm{g} / \mathrm{mL}$ group; (3) METH $296 \mu \mathrm{g} / \mathrm{mL}$ group; (4) METH $444 \mu \mathrm{g} / \mathrm{mL}$ group; (5) METH $667 \mu \mathrm{g} / \mathrm{mL}$ group group; (6) METH $1000 \mu \mathrm{g} / \mathrm{mL}$ group.
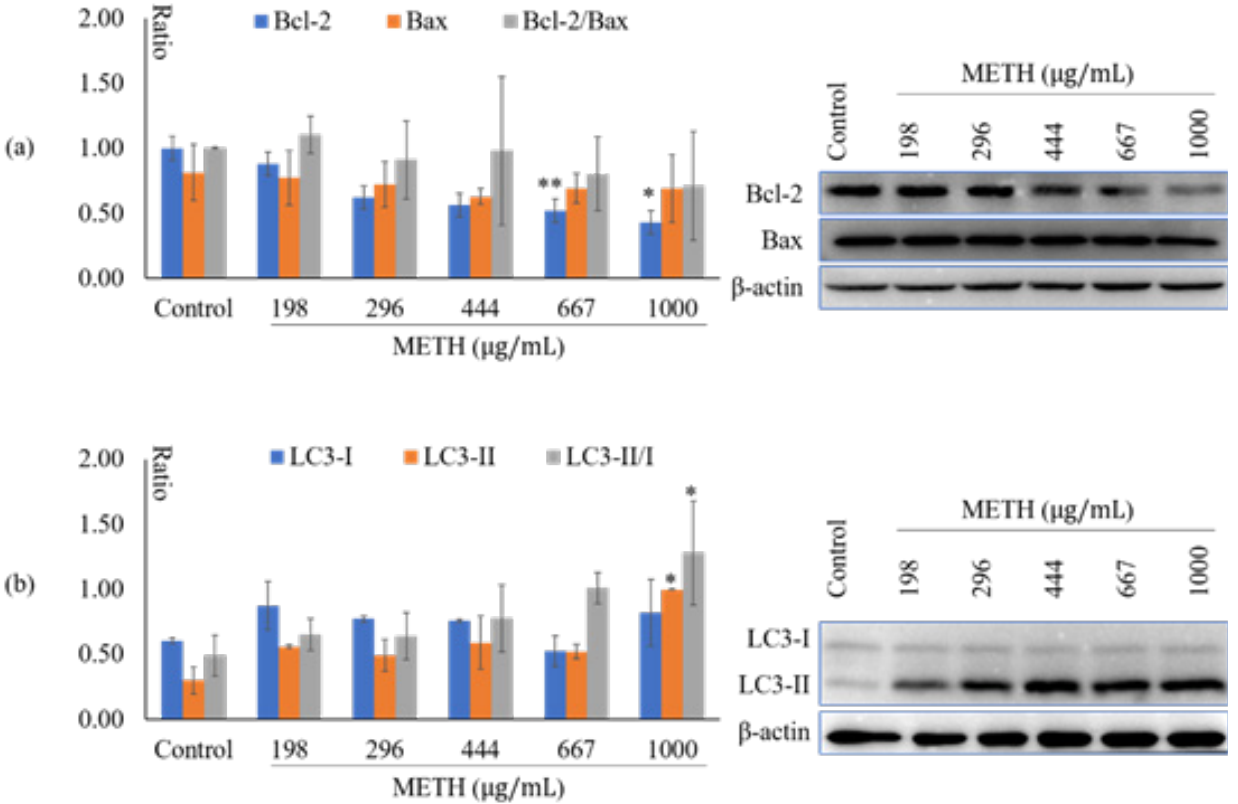

Figure 4: METH induced apoptosis and autophagy of U-118 MG cells. (a) the expression of Bcl-2 and Bax; (b) the expression of LC3. The annotation * indicates a $p$ value $<0.05$ versus control group. The annotation ** indicates a $p$ value $<0.01$ versus control group. 

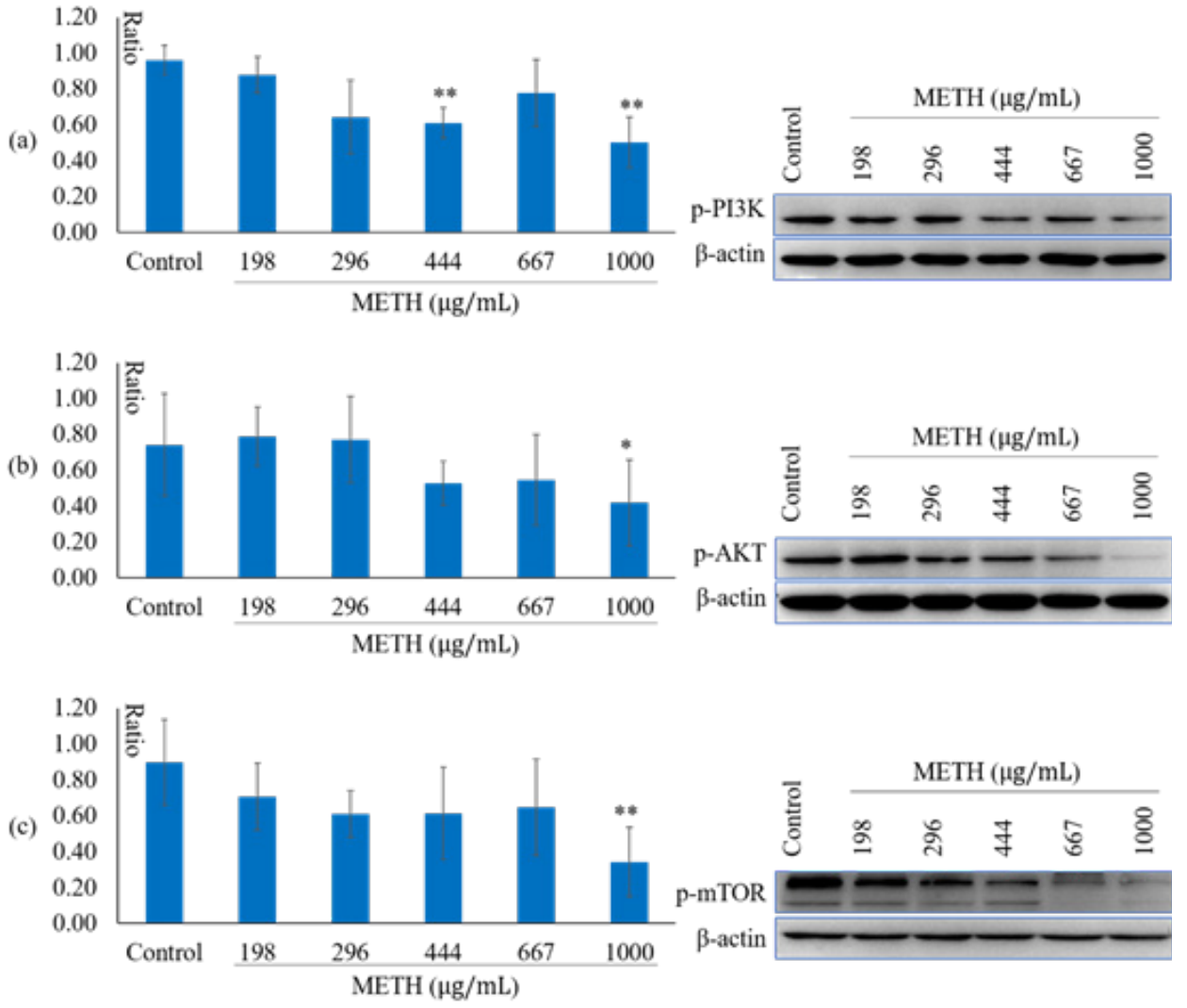

Figure 5: METH inhibited the activity of PI3K-Akt-mTOR signaling pathway. (a) the expression of p-PI3K; (b) the expression of p-Akt; (3) the expression of $p$-mTOR. The annotation * indicates a $p$ value $<0.05$ versus control group. The annotation ** indicates a $p$ value $<0.01$ versus control group.

exposure to METH (Figure 4b). These results indicated that METH induced both apoptosis and autophagy of U-118 MG cells.

To reveal the role of the PI3K-Akt-mTOR signaling pathway, we measured the phosphorylation levels of these kinases after METH treatment. The results showed that METH correlated with dephosphorylation of PI3K (Figure 5a), Akt (Figure 5b) and mTOR (Figure 5c), where PI3K and mTOR are kinases upstream and downstream of Akt, respectively. It is suggested that METH inhibited the activation of this signaling pathway.

Further, we observed the changes of kinases downstream of the mTOR complex-1 (mTORC1), including p70s6k, rps6, 4EBP1 and eIF4E. Here, the phosphorylation cascades were found to be inhibited after exposure to METH (Figure 6 a,b,c,d).

\section{Discussion}

METH, a commonly abused illegal psychostimulant, has been shown to induce neurotoxicity via multiple mechanisms, likely involving reactive oxygen species
(ROS), leading to cell death through the apoptotic pathway, disturbances in mitochondrial function, neuroinflammation and autophagy [13-15]. Microglial activation and death have been well documented to involve in neuroplasticity and drug addiction. However, effects of METH on gliacytes and the roles of the PI3K-AktmTOR signaling pathway are still unclear.

In this research, we found that METH dosedependently inhibited cell proliferation of the glial cell line U-118 MG, and induced its apoptosis and autophagy. The Bcl-2 family is one of the foremost oncogene families involved in cell apoptosis. Expression of Bcl-2 inhibits cell death by decreasing cellular generation of ROS. Our results showed that treatment of U-118 MG cells with METH reduced the expression of Bcl-2. Previous studies have shown that METH inhibits the expression of Bcl-2 in various cells (SH-SY5Y, PC12, etc.), which is consistent with our results [16-19]. These results provide potential mechanisms and help to explain METH-induced cell apoptosis.

The PI3K-Akt-mTOR signaling pathway has been demonstrated to be involved in addiction and cue-induced relapse related to several substances. In addition, this 

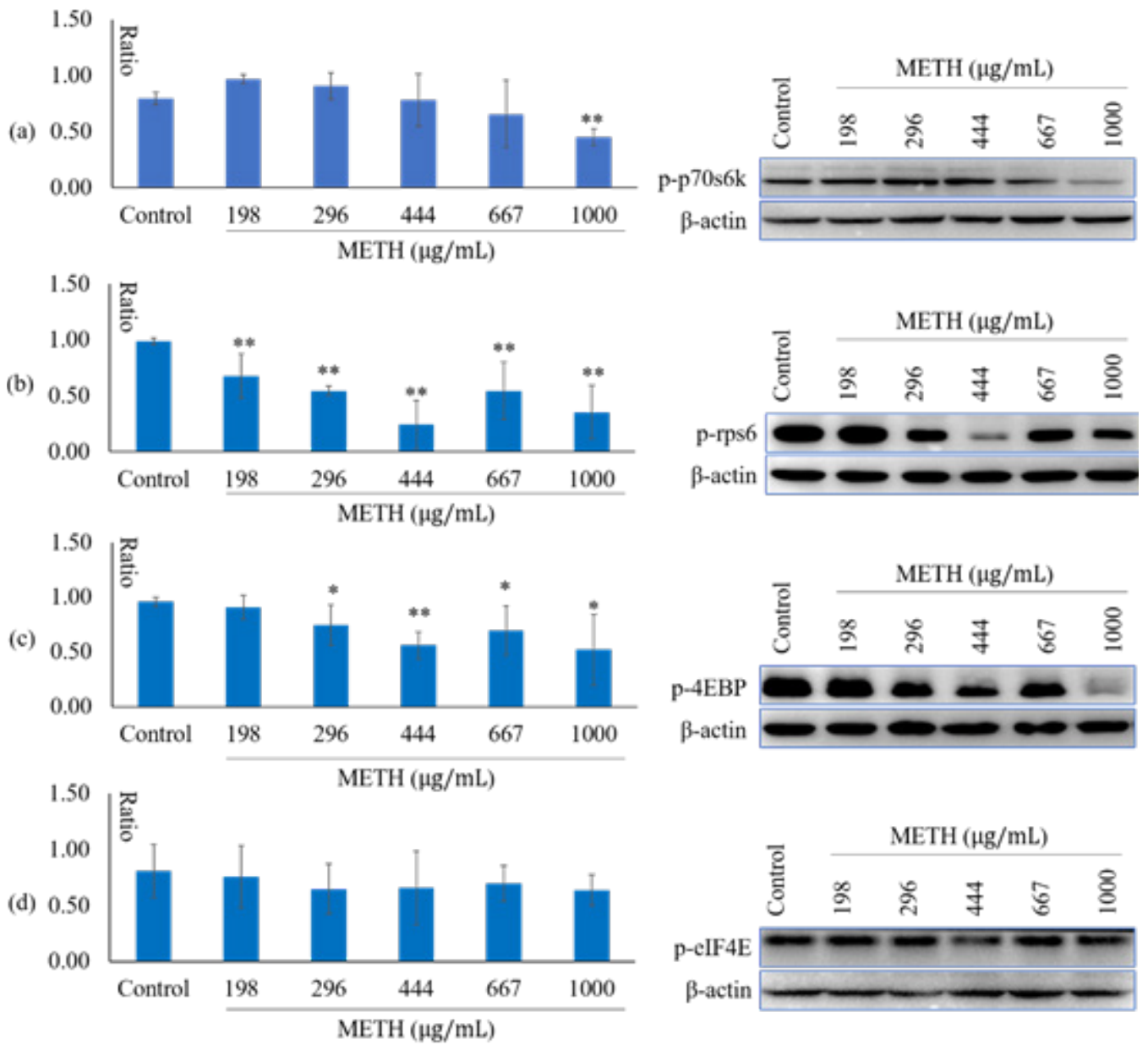

Figure 6: METH inhibited phosphorylation of downstream kinases of mTORC1. (a) the expression of p-p70s6k; (b) the expression of p-rps6; (3) the expression of $\mathrm{p}-4 \mathrm{EBP}$; (3) the expression of $\mathrm{p}$-elF4E. The annotation * indicates a $p$ value $<0.05$ versus control group. The annotation ** indicates a $p$ value $<0.01$ versus control group.

pathway is well known to regulate biological processes, including cell survival, proliferation, apoptosis and autophagy. Wu et al. [20] found that exposure to METH decreased phosphorylation within the Akt-GSK3 $\beta$-mTOR pathway in PC12 cells. Lithium protected against METHinduced neurotoxicity by reversing the dephosphorylation of Akt-GSK33-mTOR, and LY294002, a PI3K-Akt inhibitor, was found to suppress the protective effects of lithium. Lv et al. [21] found that thioredoxin-1, an important intra- and extra-cellular regulator of redox reactions, was induced by METH in PC12 cells and that the PI3K-Akt pathway was involved in the METH-induced increase of thioredoxin-1 expression.

These studies indicate that a variety of different mechanisms link the PI3K-Akt-mTOR pathway to METHinduced cell apoptosis. Our results show that exposure to METH also causes changes in phosphorylation of PI3KAkt-mTOR in U-118 MG cells, which may be associated with the cell apoptosis induced by METH in these cells.
In terms of regulating protein synthesis, the downstream effectors of mTORC1 are mainly p70s6k, rps6, 4EBP and eIF4E. Autophagy might mediate neuroprotective effects following some types of brain damage, including METH intoxication. However, autophagy precipitates a peculiar form of cell death that contributes to some types of brain damage in other circumstances [22-24]. What is clear about autophagy for now is that it is regulated by the mTOR signaling pathway. However, the potential contribution of autophagy to METH-induced damage of neuronal cells remains unclear.

There is abundant evidence that MTORC1 and its downstream kinases participate in METH-induced glial autophagy and apoptosis. For example, Li et al. [25] found that METH elevated expression of LC3-II, a protein associated with the autophagosome, in PC 12 cells, but significantly inhibited $p$-mTOR. Taurine attenuated METHinduced autophagy and apoptosis via the mTOR pathway. Hebert \& O'Callaghan [26] demonstrated that METH- 
induced glial activation in female $\mathrm{C} 57 \mathrm{BL} / 6 \mathrm{~J}$ mice was associated with significant changes in activated forms of p70s6k. Using the SK-N-SH dopaminergic cell line, Kongsuphol et al. [27] investigated the effect of METH on autophagy and its upstream regulator, the mTOR signaling pathway, and found that METH induced expression of LC3-II but inhibited phosphorylation of mTOR and 4EBP1. Huang et al. [22] confirmed that METH induces apoptosis and autophagy, and that the protective effects of L-ascorbate are mediated by inhibiting production of ROS. Ma et al. [28] reported that chronic exposure to METH disrupted cells whereas acute exposure induced early pro-survival responses mediated through inactivation of the Akt-mTOR-p70s6k pathway.

In the present study, we found that METH decreased phosphorylation of mTORC1 and its downstream kinases, but increased the LC3-II/I ratio. These results indicate that inactivation of mTORC1 and activation of autophagy following dephosphorylation of PI3K-Akt might contribute to the apoptosis of U-118 MG cells.

The effects of METH on cell proliferation or apoptosis was dose-dependently. Interestingly, the activation or inhibition of METH on protein expression and phosphorylation does not follow a strict dose-dependence as reversions were observed at the concentration of 667 $\mu \mathrm{g} / \mathrm{mL}$ for several proteins, although the overall trend was consistent. The complex nature of the dose-response relationship suggests that there are potential mechanisms other than the PI3K-Akt-mTOR signaling pathway involved in the apoptosis and autophagy induced by METH, and these alternative mechanisms deserve further discussion. Further studies should provide more direct evidence of connections, including demonstrations of the opposing effects of PI3K-Akt-mTOR agonists and inhibitors on apoptosis and direct observations of the activation of autophagy. In addition, protective effects of lithium and $\mathrm{N}$-Acetyl-L-cysteine (NAC), which was proved to influence the PI3K-Akt-mTOR signaling pathway, might be observed in further studies. Such further studies might provide a basis for the treatment of METH induced addiction and nerve injuries.

In conclusion, our results indicated that METH inhibited proliferation of U-118 MG cells and induced apoptosis and autophagy in this cell line and that the PI3K-Akt-mTOR signaling pathway might play critical roles in these effects.

Acknowledgements: We thank the Shenzhen Science and Technology Planning Project (JCYJ20160427185055877, JCYJ20160429090753103, JCYJ20170412171856582), the Health and Family Planning Commission of
Shenzhen (201605018), Shenzhen "Sanming” project (SZSM201512026), Guangdong Bureau of Traditional Chinese Medicine Project (20171228) and Guangdong Medical Research Project (B2018090) for financial support and the Compulsory Isolation and Detoxification Program of Shenzhen for kindly providing the METH.

Disclosure Statement: The authors declare no potential conflicts of interest with respect to the research, authorship, and/or publication of this article.

\section{References}

[1] Dang D.K., Shin E.J., Nam Y., Ryoo S., Jeong J.H., Jang C.G., et al., Apocynin prevents mitochondrial burdens, microglial activation, and pro-apoptosis induced by a toxic dose of methamphetamine in the striatum of mice via inhibition of p47phox activation by ERK, J Neuroinflammation., 2016, 13, 12.

[2] Li F.C., Yen J.C., Chan S.H., Chang A.Y., Bioenergetics failure and oxidative stress in brain stem mediates cardiovascular collapse associated with fatal methamphetamine intoxication, PloS one., 2012, 7, e30589.

[3] Qie X., Wen D., Guo H., Xu g., Liu S., Shen Q., et al., Endoplasmic Reticulum Stress Mediates Methamphetamine-Induced BloodBrain Barrier Damage, Front Pharmacol., 2017, 8, 639.

[4] Venkatesan A., Uzasci L., Chen Z., Rajbhandari L., Anderson C., Lee M.H., et al., Impairment of adult hippocampal neural progenitor proliferation by methamphetamine: role for nitrotyrosination, Mol Brain., 2011, 4, 28.

[5] Shen K., Zhang Y., Lv X., Chen X., Zhou R., Nguyen LK., et al., Molecular Mechanisms Involving Sigma-1 Receptor in Cell Apoptosis of BV-2 Microglial Cells Induced by Methamphetamine, CNS Neurol Disord Drug Targets., 2016, 15, 857-865.

[6] Chao J., Zhang Y., Du L., Zhou R., Wu X., Shen K., et al., Molecular mechanisms underlying the involvement of the sigma-1 receptor in methamphetamine-mediated microglial polarization, Sci Rep., 2017, 7, 11540.

[7] Ersahin T., Tuncbag N., Cetin-Atalay R., The PI3K/AKT/mTOR interactive pathway, Mol Biosyst., 2015, 11, 1946-1954.

[8] Hermida M.A., Dinesh Kumar J., Leslie N.R., GSK3 and its interactions with the PI3K/AKT/mTOR signalling network, Adv Biol Regul., 2017, 65, 5-15.

[9] O’Donnell J.S., Massi D., Teng M.W.L., Mandala M., PI3K-AKTmTOR inhibition in cancer immunotherapy, redux, Semin Cancer Biol., 2018, 48, 91-103.

[10] Neasta J., Barak S., Hamida S.B., Ron D., mTOR complex 1: a key player in neuroadaptations induced by drugs of abuse, J Neurochem., 2014, 130, 172-184.

[11] Luo Y.X., Han H., Shao J., Gao Y., Yin X., Zhu W.L., et al., mTOR signalling in the nucleus accumbens shell is critical for augmented effect of TFF3 on behavioural response to cocaine, Sci Rep., 2016, 6, 27895.

[12] Shi Y., Wang F., Hu A.Z., Wang Q.W., Wu J.L., Li M.H., Effects and Mechanisms of Jinniu Capsule on Methamphetamine-Induced Conditioned Place Preference in Rats, Open Chem., 2018, 16, 674-680. 
[13] Bortell N., Najera J.A., Sanchez-Alavez M., Marcondes MC., In vivo effects of methamphetamine on brown fat reactive oxygen species and mitochondria, Temperature (Austin)., 2015, 2, 453.

[14] Zhang Y., Shen K., Bai Y., Lv X., Huang R., Zhang W., et al., Mir143$B B C 3$ cascade reduces microglial survival via interplay between apoptosis and autophagy: Implications for methamphetaminemediated neurotoxicity, Autophagy., 2016, 12, 1538-1559.

[15] Roohbakhsh A., Shirani K., Karimi G., Methamphetamineinduced toxicity: The role of autophagy?, Chem Biol Interact., 2016, 260, 163-167.

[16] Zhu J., Zang S., Chen X., Jiang L., Gu A., Cheng J., et al., Involvement of the delayed rectifier outward potassium channel Kv2.1 in methamphetamine-induced neuronal apoptosis via the p38 mitogen-activated protein kinase signaling pathway, J Appl Toxicol., 2018, 38, 696-704.

[17] Zhao J., Liu J., Xu E., Liu Y., Xie A., Xiong H., dl-3-n-Butylphthalide attenuation of methamphetamine-induced neurotoxicity in SH-SY5Y neuroblastoma cells, Life Sci., 2016, 165, 16-20.

[18] Chetsawang J., Suwanjang W., Pirompul N., Govitrapong P., Chetsawang B., Calpastatin reduces methamphetamineinduced induction in c-Jun phosphorylation, Bax and cell death in neuroblastoma SH-SY5Y cells, Neurosci Lett., 2012, 506, 7-11.

[19] Coelho-Santos V., Goncalves J., Fontes-Ribeiro C., Silva A.P., Prevention of methamphetamine-induced microglial cell death by TNF-alpha and IL- 6 through activation of the JAK-STAT pathway, J Neuroinflammation., 2012, 9, 103.

[20] Wu J., Zhu D., Zhang J., Li G., Liu Z., Sun J., Lithium protects against methamphetamine-induced neurotoxicity in PC12 cells via Akt/GSK3beta/mTOR pathway, Biochem Biophys Res Commun., 2015, 465, 368-373.

[21] Lv T., Wang S.D., Bai J., Thioredoxin-1 was required for CREB activity by methamphetamine in rat pheochromocytoma cells, Cell Mol Neurobiol., 2013, 33, 319-325.

[22] Huang Y.N., Yang L.Y., Wang J.Y., Lai C.C., Chiu C.T., Wang J.Y., L-Ascorbate Protects Against Methamphetamine-Induced Neurotoxicity of Cortical Cells via Inhibiting Oxidative Stress, Autophagy, and Apoptosis, Mol Neurobiol., 2017, 54, 125-136.

[23] Wongprayoon P., Govitrapong P., Melatonin Attenuates Methamphetamine-Induced Neurotoxicity, Curr Pharm Des., 2016, 22, 1022-1032.

[24] Li B., Chen R., Chen L., Qiu P., Ai X., Huang E., et al., Effects of DDIT4 in Methamphetamine-Induced Autophagy and Apoptosis in Dopaminergic Neurons, Mol Neurobiol., 2017, 54, 1642-1660.

[25] Li Y., Hu Z., Chen B., Lu W., Deng Y., Zhu R., et al., Taurine attenuates methamphetamine-induced autophagy and apoptosis in PC12 cells through mTOR signaling pathway, Toxicol Lett., 2012, 215, 1-7.

[26] Hebert M.A., O'Callaghan J.P., Protein phosphorylation cascades associated with methamphetamine-induced glial activation, Ann N Y Acad Sci., 2000, 914, 238-262.

[27] Kongsuphol P., Mukda S., Nopparat C., Villarroel A., Govitrapong P., Melatonin attenuates methamphetamine-induced deactivation of the mammalian target of rapamycin signaling to induce autophagy in SK-N-SH cells, J Pineal Res., 2009, 46, 199 206.

[28] Ma J., Wan J., Meng J., Banerjee S., Ramakrishnan S., Roy S., Methamphetamine induces autophagy as a pro-survival response against apoptotic endothelial cell death through the Kappa opioid receptor, Cell Death Dis., 2014, 5, e1099. 